\title{
Cerebral Histologic and Electrocorticographic Changes after Asphyxia in Fetal Sheep
}

\author{
A. J. GUNN, J. T. PARER, E. C. MALlaRD, C. E. WILliams, AND P. D. GLUCKMAN
}

Developmental Physiology Laboratory, Department of Paediatrics, University of Auckland, New Zealand [A.J.G.,

E.C.M., C.E.W., P.D.G.j; and Department of Obstetrics, Gynecology, and Reproductive Sciences and

Cardiovascular Research Institute, University of California, San Francisco, California 94143-0132 [J.T.P.]

\begin{abstract}
Asphyxia can cause neurologic damage in the fetus, but there are few data relating severity or duration of asphyxia to the degree of cerebral damage. We report cerebral histologic and electrophysiologic changes after asphyxia in chronically instrumented late-gestation fetal sheep. We reduced uterine blood flow to produce an ascending aortic blood oxygen content $<1.5 \mathrm{mM}$ for either 30 or $60 \mathrm{~min}(n=13)$. In a subsequent protocol $(n=6)$, if full occlusion of the common uterine artery for 15 min did not reduce the EEG voltage to less than $20 \%$ of baseline, supplementary maternal hypoxia was added for a maximum of 120 min. Histologic outcome was assessed 3 d postinsult. Uterine artery occlusion resulted in severe hypoxemia, hypercarbia, acidosis, and an initial hypertension and bradycardia. Eight of 14 surviving fetuses showed neuronal damage, with greatest loss in the parasagittal cortex, striatum, and the $\mathrm{CA}^{1 / 2} / 2$ region of the hippocampus. Neuronal damage was strongly associated with the percentage of decrease in blood pressure during the insult $(r=0.75, p<0.005)$ but not with the degree of hypoxia. No other factor was independently predictive, but, when considered separately, $\mathrm{pH}(r=0.54 ; p<0.05)$ and loss of intensity of the EEG $(r=0.61, p<0.02)$ at the end of asphyxia were also correlated with outcome. The $\mathrm{pH}$ fell to $<7.0$ in six of eight fetuses with damage, whereas it remained $>7.0$ in five of six without damage $(p<0.05)$. We conclude that severe intrauterine asphyxia for periods of 30 to $120 \mathrm{~min}$ can cause predominant parasagittal neuronal death and that this is associated with hypotension, severe metabolic acidosis, and suppression of EEG during the insult. These data are consistent with the suggestion that impairment of cerebral perfusion is a critical event in localizing cerebral damage during perinatal asphyxia. ( $P e$ diatr Res 31: 486-491, 1992)
\end{abstract}

\section{Abbreviations}

$\mathrm{PaO}_{2}$, arterial $\mathrm{O}_{2}$ pressure

$\mathrm{PaCO}_{2}$, arterial $\mathrm{CO}_{2}$ pressure

BP, blood pressure

$\mathrm{CaO}_{2}$, arterial $\mathrm{O}_{2}$ content

Perinatal asphyxia affects approximately six of 1000 newborn infants (1) and is associated with neurologic damage or death (2). It is believed to occur prenatally in many cases $(3,4)$, but there are few data relating severity or duration of fetal asphyxia

Received October 10, 1991; accepted December 19, 1991.

Correspondence: A. J. Gunn, Developmental Physiological Laboratory, Department of Paediatrics, University of Auckland, Private Bag, Auckland, New Zealand.

Supported by grants from the Medical Research Council of New Zealand, the Neurological Foundation, and the Auckland Medical Research Foundation. to the degree of cerebral damage $(5,6)$. Indeed, there remains considerable controversy regarding the factors that contribute to perinatal hypoxic ischemic encephalopathy. We therefore examined the histologic and electrophysiologic changes associated with asphyxia due to uterine artery occlusion of varying duration in fetal sheep.

\section{MATERIALS AND METHODS}

Pregnant sheep from 119 to $128 \mathrm{~d}$ of gestation (term is $147 \mathrm{~d}$ ) were instrumented under $2 \%$ halothane anesthesia with an adjustable occluder, made from Teflon-lined cardiac catheterization introducers and catheters, around the maternal common uterine artery. The ovarian arteries were ligated just proximal to the ovaries to prevent anastomotic perfusion. The fetus was then instrumented as previously described $(7,8)$. Polyvinyl catheters were inserted into fetal ascending aortic arteries and the amniotic cavity. Two pairs of shielded stainless steel recording electrodes were placed over the parietal dura, $10 \mathrm{~mm}$ lateral to bregma, and $5 \mathrm{~mm}$ and $15 \mathrm{~mm}$ anterior. In seven fetuses, a pair of stimulating electrodes was also placed $15 \mathrm{~mm}$ lateral and $10 \mathrm{~mm}$ anterior to bregma. Electrocardiographic electrodes were placed s.c. over the shoulders. In the first five preparations, an electromagnetic flow transducer was placed on a branch of the uterine artery. This was subsequently discontinued because in all five fetuses the flow was essentially zero during the study and bore no relationship to the fetal condition. Residual flow to the uterus was assumed to be via unligated ovarian vessels.

All studies were performed 4 or more d after surgery. The EEG from 1 to $30 \mathrm{~Hz}$ was recorded and its power spectrum analyzed in real time (7) for a minimum of $4 \mathrm{~h}$ preocclusion and for $72 \mathrm{~h}$ afterwards. In seven fetuses, additionally, changes in the electrical impedance of the parasagittal cortex were measured using a fourelectrode technique, as previously described (8). Fetal arterial $\mathrm{BP}$, corrected for amniotic fluid pressure, fetal heart rate, and uterine blood flow were recorded for $1 \mathrm{~h}$ before the occlusion. Blood samples were analyzed for $\mathrm{pH}, \mathrm{PaCO}_{2}, \mathrm{PaO}_{2}$, arterial $\mathrm{O}_{2}$ saturation, $\mathrm{Hb}$, and lactate $30 \mathrm{~min}$ and $2 \mathrm{~min}$ before occlusion and then every $15 \mathrm{~min}$ during the occlusion. In addition, specimens were taken $5 \mathrm{~min}$ after occlusion and as required by the fetal condition for $\mathrm{pH}$ and blood gases.

Twenty-two fetuses were investigated, using two protocols; three fetuses that died from proven infection were excluded from analysis (Table 1). The fetuses were $125 \pm 4 \mathrm{~d}$ old at the time of the insult, and their postmortem weights $(2.69 \pm 0.45 \mathrm{~kg})$ and biparietal diameters $(5.4 \pm 0.2 \mathrm{~cm})$ were normal by the standards of our laboratory and were not significantly different between the outcome groups. Thirteen fetuses underwent protocol A, in which the uterine artery occluder was progressively tightened over a period of 5 to $10 \mathrm{~min}$, to produce $\mathrm{a} \mathrm{CaO}_{2}$ of $<1.5 \mathrm{mM}$, inasmuch as this degree of hypoxemia is associated with altered oxidative cerebral metabolism (9). After either 30 or $60 \mathrm{~min}$, the 
Table 1. Incidence of neuronal damage vs protocol

\begin{tabular}{lcccc}
\hline & \multicolumn{2}{c}{ Protocol A } & & \\
\cline { 2 - 3 } \multicolumn{1}{c}{ Outcome } & $30 \mathrm{~min}$ & $45-60 \mathrm{~min}$ & Protocol B & Totals \\
\hline No damage & 3 & 3 & 0 & 6 \\
<10\% damage & 1 & 3 & 1 & 5 \\
>10\% damage & 0 & 1 & 2 & 3 \\
Fetus died & 2 & 0 & 3 & 5 \\
Total & 6 & 7 & 6 & 19 \\
\hline
\end{tabular}

occluder was released and the above fetal variables monitored for a further $60 \mathrm{~min}$.

Because in protocol A we found that only fetuses with significant flattening of the EEG during asphyxia developed cerebral damage, in an additional six fetuses the above approach was modified as follows (protocol B): If after $15 \mathrm{~min}$ of complete uterine artery occlusion the EEG voltage was not reduced to $<20 \%$ of baseline, inhalational hypoxia was instituted by fitting a plastic bag over the ewe's head and feeding in a $\mathrm{N}_{2} / \mathrm{O}_{2}$ mix at $20 \mathrm{~L} / \mathrm{min}$. This was required in four of six cases. The initial mixture contained $12 \% \mathrm{O}_{2}$, but it was then adjusted as appropriate to produce sustained flattening of the EEG. Each experiment was terminated at the shortest of I) 120 min of asphyxia, 2) $60 \mathrm{~min}$ of flattened EEG, or 3) if the fetus, after a period of stable asphyxia, developed a persistent bradycardia $<60 \mathrm{bpm}$ or a profound fall in BP.

Histology. When the fetuses were killed at $72 \mathrm{~h}$ postasphyxia, histologic sections of the brains were obtained as previously described (7); no histology was performed on the five fetuses that died during or shortly after the insult. Briefly, the brain was perfused through the common carotid arteries with saline followed by $10 \%$ formalin, embedded in paraffin, coronally subserially sectioned to $8 \mu \mathrm{M}$, and then stained with thionine/acid fuchsin (10). Each section was examined by light microscopy by two assessors, one of whom was blind to the original study. Each region was scored in a number of preassigned areas for the proportion of neurons with ischemic cell change, with acidophilic (red) cytoplasm and contracted nuclei, or with just a thin rim of red cytoplasm with pyknotic nuclei. Such cells were assessed as dead, whereas all others were considered viable. The damage scores were as follows: $0=$ no dead neurons, $5=>0-10 \%, 30=$ $>10-50 \%, 70=>50-90 \%, 95=90-99 \%$, and $100=100 \%$ dead neurons. Each score corresponds with the midpoint of its range.

Analysis. The integrated oxygen deficit was calculated as the area under the curve of the difference between the preinsult ascending aortic oxygen content and that at each time point, against time in minutes. The EEG intensity was presented in $\mathrm{dB}$ $[\log (\mathrm{x}) \cdot 10]$, because this transformation gives a better approximation to the normal distribution (11). The intensity was then normalized with respect to the 4-h control period; thus, all measurements are expressed as a ratio of the baseline period. Time series analysis of both the EEG and impedance were based on data smoothed with a digital Blackman low-pass filter with a cutoff of $0.25 \mathrm{~Hz}$ to eliminate short-term $(<10 \mathrm{~min})$ fluctuations. The final EEG intensity was calculated on the final 4-h period, from 68 to $72 \mathrm{~h}$. The median frequency of the EEG was calculated on the saved power spectrum at four periods: the 4-h control, during the occlusion, from 5 to $30 \mathrm{~h}$, and the final 4-h recovery period. The recovery time of the EEG was defined as the time postinsult that the EEG was more than $5 \mathrm{~dB}$ below the baseline intensity. The impedance was expressed as a percentage of baseline levels and reflects changes in extracellular space (8).

For analysis of outcome, fetuses were grouped as follows: 1$)$ no damage in the parasagittal cortex, 2) mild damage $(<10 \%$ neuronal loss), 3) moderate to severe loss (>10\% neuronal loss) and 4) death. Comparisons of EEG and biochemical parameters were made by two-way analysis of variance for outcome group, with time as a repeated measure. Where significant differences were found, post hoc comparisons of the means were made using the Neuman-Keuls test, adjusted using the baseline levels as a covariate, or the paired $t$ test with the level of significance adjusted using the Bonferroni method. The relationship between the parasagittal damage scores and the changes in various parameters was further analyzed by stepwise multiple regression. Fisher's exact test was used to test proportions.

\section{RESULTS}

The overall outcome of the protocols is shown in Table 1. The duration of occlusion was not significantly related to outcome. Eight of the 14 surviving fetuses showed cerebral damage, with neuronal death greatest in the parasagittal cortex, usually with accentuation in the sulci, the striatum, and the $\mathrm{CA}^{1 / 2}$ region of the hippocampus. The hippocampus was less frequently affected than the cortex, with neuronal loss in only four fetuses $(p<$ $0.05)$. The lateral (temporal) cortex, dentate gyrus, thalamus, and amygdala were relatively spared $(p<0.05$, Fig. 1$)$.

Five fetuses died, three during the insult. Immediate release of uterine occlusion and the administration of intraarterial adrenaline were not sufficient to save these fetuses once the precipitous fall in BP and heart rate was noted. Two fetuses died several hours after the end of the occlusion, after demonstrating full recovery of the $\mathrm{PaO}_{2}$ and partial recovery of the EEG and base deficit. In both cases, postmortem amniotic fluid culture was negative.

Uterine artery occlusion resulted in hypoxemia, hypercarbia, acidosis, and an initial hypertension and bradycardia. The changes in $\mathrm{pH}, \mathrm{CaO}_{2}$, base excess, and $\mathrm{BP}$, related to outcome, are shown in Tables 2 and 3. The baseline biochemical parameters were normal in each of the four outcome groups, as were the 72-h values (data not shown).

More severe acidosis was seen at the end of occlusion in fetuses with neuronal loss, arterial $\mathrm{pH}<7.0$ in six of eight $(6.9 \pm 0.1)$

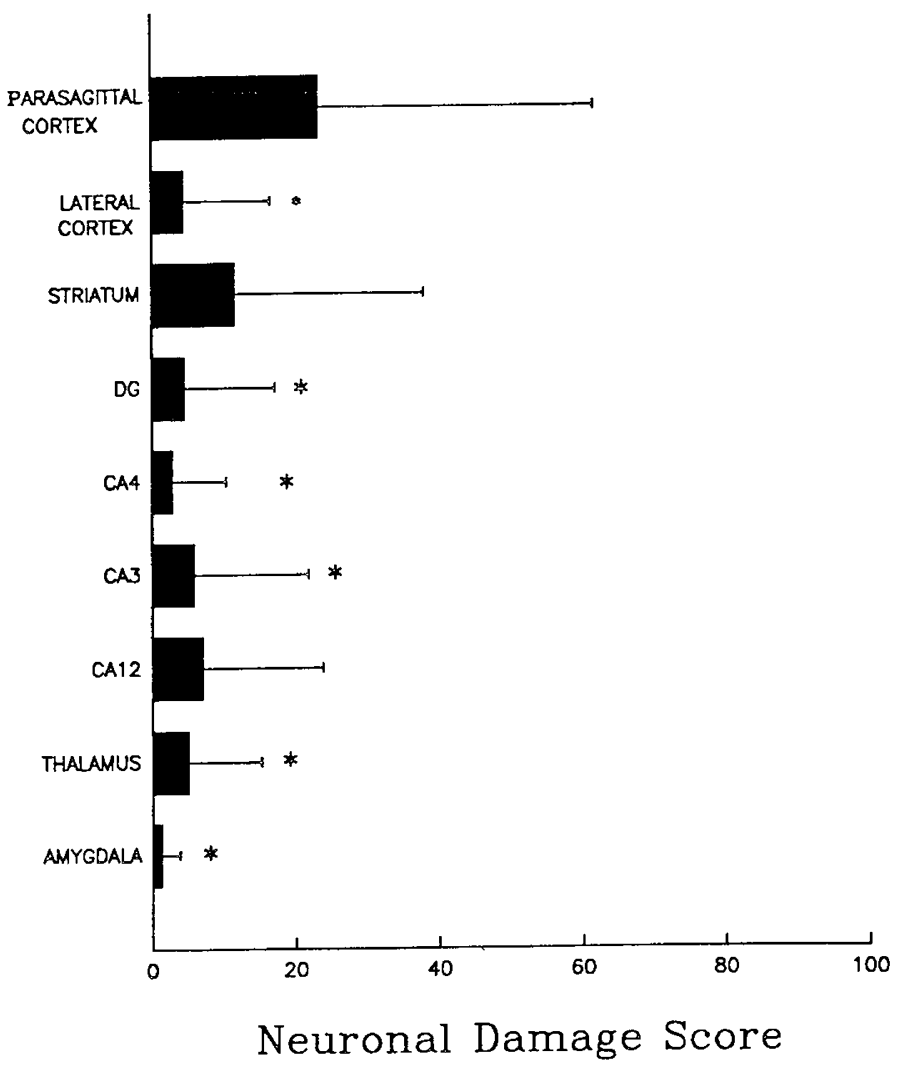

Fig. 1. Distribution of neuronal damage in the eight surviving fetuses with neuronal loss. Neuronal death is greatest in the parasagittal cortex and the striatum. $D G$ (dentate gyrus), $C A 4, C A 3$, and $C A 12$ are regions of the hippocampus. ${ }^{*}, p<0.05$ compared with the parasagittal cortex. 
Table 2. Changes in fetal arterial blood gases and $p H$ vs outcome of asphyxia in utero

\begin{tabular}{|c|c|c|c|c|}
\hline & \multicolumn{4}{|c|}{ Outcome (mean $\pm \mathrm{SD})$} \\
\hline & No damage & $<10 \%$ damage & $>10 \%$ damage & Fetus died \\
\hline \multicolumn{5}{|l|}{$\mathrm{pH}$} \\
\hline Terminal* & $7.12 \pm 0.09 \dagger$ & $6.95 \pm 0.15 \dagger \ddagger$ & $6.85 \pm 0.05 \dagger \ddagger$ & $6.77 \pm 0.07 \dagger \ddagger$ \\
\hline Recovery§ & $7.25 \pm 0.07 \dagger$ & $7.14 \pm 0.09 \dagger$ & $7.08 \pm 0.08 \dagger \ddagger$ & $7.06 \pm 0.18 \dagger$ \\
\hline \multicolumn{5}{|c|}{ Base excess $(\mathrm{mmol} / \mathrm{L})$} \\
\hline Recovery§ & $-3.9 \pm 4.7 \dagger$ & $-11.2 \pm 5.3 \dagger$ & $-13.6 \pm 4.6+\ddagger$ & $-14.7 \pm 7.9 \dagger \ddagger$ \\
\hline \multicolumn{5}{|l|}{$\mathrm{CaO}_{2}(\mathrm{mmol} / \mathrm{L})$} \\
\hline Basal & $3.3 \pm 1.2$ & $3.1 \pm 0.9$ & $3.6 \pm 0.5$ & $3.2 \pm 1.1$ \\
\hline Minimum & $0.84 \pm 0.43 \dagger$ & $0.55 \pm 0.54 \dagger$ & $0.85 \pm 0.19 \dagger$ & $0.66 \pm 0.34 \dagger$ \\
\hline Mean? & $0.99 \pm 0.42 \dagger$ & $0.85 \pm 0.54 \dagger$ & $1.26 \pm 0.36 \dagger$ & $0.96 \pm 0.56 \dagger$ \\
\hline Recovery§ & $2.97 \pm 1.19$ & $2.95 \pm 0.91$ & $2.51 \pm 0.59$ & $2.68 \pm 0.75$ \\
\hline $\mathrm{ICaO}_{2}{ }^{* *}$ & $253 \pm 152$ & $239 \pm 136$ & $225 \pm 58$ & $359 \pm 183$ \\
\hline
\end{tabular}

* At the end of asphyxia.

$\dagger p<0.01$ vs baseline period.

$\ddagger p<0.05 v s$ nil damage.

$\S$ One $\mathrm{h}$ postasphyxia; $n=2$ for fetuses that died, at this time point.

$\| p<0.05$ vs survivors with damage.

If Values recorded during asphyxia.

** $\mathrm{ICaO}_{2}$, integrated $\mathrm{CaO}_{2}$ deficit (mmol.min).

Table 3. Fetal arterial $\mathrm{BP}(\mathrm{kPa})$ vs outcome

\begin{tabular}{llllc}
\hline & \multicolumn{4}{c}{ Outcome (mean \pm SD) } \\
\cline { 2 - 5 } & \multicolumn{1}{c}{$\begin{array}{c}\text { No } \\
\text { damage }\end{array}$} & $\begin{array}{c}<10 \% \\
\text { damage }\end{array}$ & $\begin{array}{c}>10 \% \\
\text { damage }\end{array}$ & Fetus died \\
\hline Basal BP & $6.1 \pm 1.1$ & $5.7 \pm 0.6$ & $5.8 \pm 0.3$ & $6.2 \pm 0.4$ \\
Minimum BP* & $6.4 \pm 0.9$ & $5.0 \pm 0.8 \dagger \ddagger$ & $3.5 \pm 0.8 \dagger \ddagger$ & $2.6 \pm 1.1 \dagger \ddagger \S$ \\
Mean BP* & $7.0 \pm 1.0 \ddagger$ & $6.2 \pm 1.1$ & $5.1 \pm 0.6 \dagger \ddagger$ & $4.5 \pm 0.8 \dagger \ddagger \|$ \\
Recoveryף & $6.6 \pm 0.6$ & $6.0 \pm 0.6$ & $5.5^{* *}$ & $6.0^{* *}$ \\
\hline
\end{tabular}

* Values recorded during asphyxia.

$\dagger p<0.05$ vs no damage.

$\ddagger p<0.01$ vs baseline values.

$\S p<0.05$ vs survivors with damage.

$\| p<0.05$ vs $<10 \%$ damage.

I One $\mathrm{h}$ postasphyxia.

** $n=1$.

versus $>7.0(7.12 \pm 0.1)$ in five of six without damage, $p<0.05$ (Table 2). The metabolic component of the acidosis, but not the respiratory component, showed a strong relationship with outcome $(p<0.003$, Table 2$)$. The greatest base deficit occurred in the fetuses that died and the least occurred in those with no damage, whereas survivors with damage showed intermediate values. Most of the deficit was accounted for by lactate, with an average rise from $2.2 \pm 1.2 \mathrm{mM}$ preinsult to $12.4 \pm 3.7 \mathrm{mM}$ at the end of occlusion $(p<0.001)$. There was only minimal recovery in the base deficit or lactate levels by 1 postinsult (lactate $=10.5 \pm 3.4 \mathrm{mM}$ ). In parallel with the rise in lactate, there was a small rise in blood glucose, from $1.0 \pm 0.3$ to $1.5 \pm 0.5 \mathrm{mM}$ $(p<0.01)$, which was not significantly related to outcome.

In contrast, while the $\mathrm{PaCO}_{2}$ rose to a peak of $12.9 \pm 4.1 \mathrm{kPa}$ at $35.5 \pm 23.3 \mathrm{~min}$, with a small fall of $0.9 \pm 1.6 \mathrm{kPa}(p<0.05)$ by the end of the occlusion, this was not associated with outcome.

The $\mathrm{PaO}_{2}$ fell from $2.8 \pm 0.6 \mathrm{kPa}$ to a trough of $1.4 \pm 0.3 \mathrm{kPa}$ ( $p<0.001)$, with a mean of $1.7 \pm 0.4 \mathrm{kPa}$ during the occlusion, and recovered fully by $1 \mathrm{~h}$ postinsult in survivors $(2.6 \pm 0.7$ $\mathrm{kPa}$ ). However, the magnitude of this response was similar in all groups. The changes in $\mathrm{CaO}_{2}$ during asphyxia showed the same pattern (Table 2), and the integrated oxygen deficit, which takes into account the duration of hypoxemia, was also not related to outcome.

Arterial BP during asphyxia was strongly associated with out- come (Table 3 ). The fetuses with no or mild damage showed a rise in mean arterial BP $(15.6 \pm 12.8$ and $8.8 \pm 14.7 \%$, respectively), whereas those that had moderate to severe damage showed a significant fall in mean BP $(-11 \pm 9 \%, p<0.01)$. Episodes of hypotension (defined as a fall of greater than $15 \%$ of baseline) occurred in only five surviving fetuses, all of whom had neuronal loss. The fetus with the most sustained hypotension, two thirds of baseline for $0.5 \mathrm{~h}$, had severe global cerebral necrosis. Conversely $(p<0.05)$, of the nine fetuses in whom hypotension did not occur all had either no or mild (three of nine) neuronal loss.

The reduction in intensity of the EEG during the insult was associated with outcome (Table 4). The fetuses that died or had severe damage showed the greatest suppression of the EEG during the insult $(p<0.05)$. Loss of intensity at $72 \mathrm{~h}$ was associated with neuronal loss. There was a clear separation between those with moderate to severe damage, who showed more than a 2-dB loss of intensity, and all the rest, who had either mild loss of intensity or an increase (Fig. 2). The median frequency of the EEG fell uniformly during asphyxia (Table $4, p<0.0001$ ). Surviving fetuses with moderate to severe damage show a shift toward lower frequencies after the insult, compared with both baseline and fetuses with no or mild damage, at 5 to $30 \mathrm{~h}(p<$ $0.005)$ and after $72 \mathrm{~h}(p<0.02)$.

Although the recovery time of the EEG intensity postasphyxia was associated with outcome (Table 4), the variability was large. Seizures, both early onset and delayed onset, were associated with neuronal damage $(p<0.01)$. Laminar necrosis of the underlying cortex occurred in fetuses that developed prolonged postasphyxial seizures (Fig. 3).

Cerebral impedance rose progressively during asphyxia, peaking at $115 \pm 9 \%$ of baseline between 5 and 10 min postrelease $(n=7)$, and then rapidly, to $<25 \%$ of the rise, by $60 \mathrm{~min}$ postasphyxia in six of seven fetuses. Neither the magnitude of the acute rise $(r=0.81, p<0.1)$ nor the duration of elevated impedance during asphyxia $(r=0.44, p<0.5)$ was significantly associated with neuronal loss.

The predictive power of variables known at the end of asphyxia were examined by multiple regression analysis against the parasagittal damage score. The most powerful factor was the change in BP during asphyxia, with a strong correlation of minimum arterial BP to neuronal loss $(r=-0.69, p<0.01)$; no other factor was independently predictive. The terminal $\mathrm{pH}(r=0.54, p<$ 
Table 4. EEG parameters vs outcome after asphyxia in utero

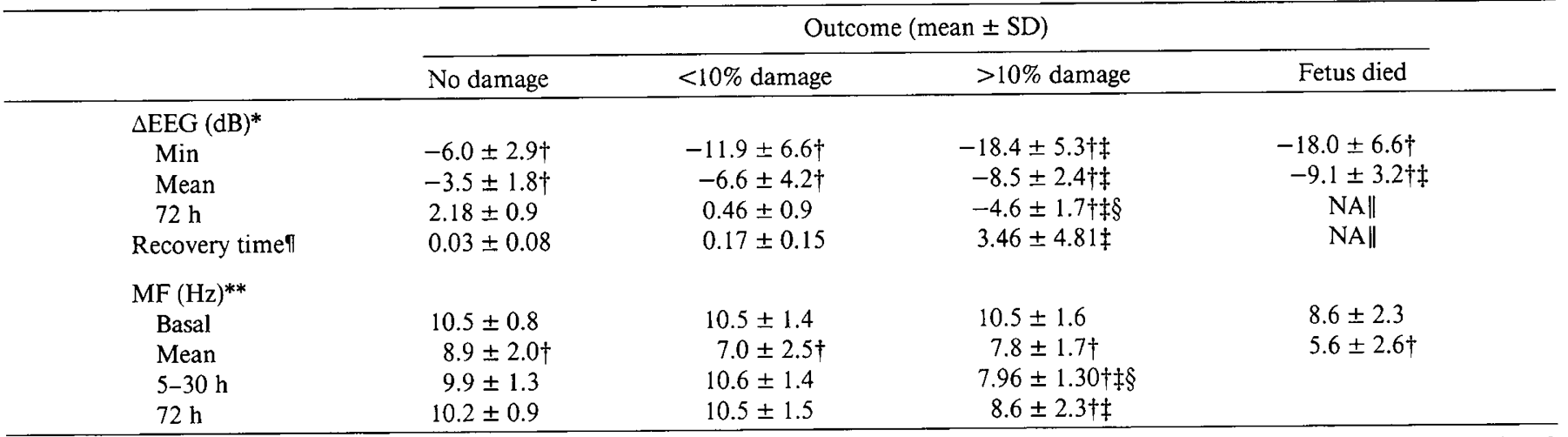

* All EEG values were recorded relative to baseline. Min, lowest value recorded during asphyxia; mean, mean EEG intensity during asphyxia; 72 $\mathrm{h}$, mean EEG intensity during a 4-h period $72 \mathrm{~h}$ postinsult.

$\dagger p<0.05 v s$ baseline period.

$\$ p<0.05$ vs no damage.

$\S p<0.05 v s<10 \%$ damage.

$\|$ Not applicable.

If Time in $\mathrm{h}$ that the EEG was more than $5 \mathrm{~dB}$ below baseline intensity after the insult.

** MF, median frequency of the EEG; mean, mean value during asphyxia. Subsequent times are postasphyxia.

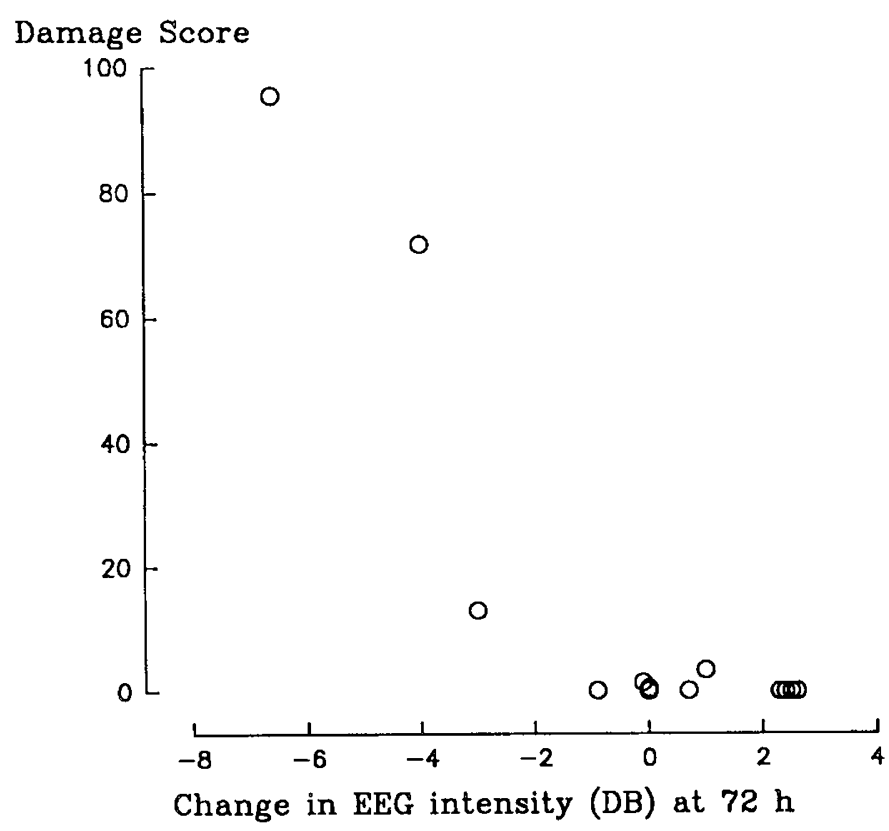

Fig. 2. EEG intensity $72 \mathrm{~h}$ postinsult $v s$ the neuronal damage score in the underlying parasagittal cortex. The EEG is normalized so that the baseline EEG is 0 . A loss of more than $2 \mathrm{db}$ in EEG intensity is associated with moderate to severe neuronal loss $(p<0.05) . D B$, decibels.

$0.05)$ and the minimum EEG intensity $(r=0.61, p<0.02)$ were both significant when considered separately, but the degree of hypoxemia was not. Postinsult, loss of EEG intensity after $3 \mathrm{~d}$ was also closely related to damage to the underlying cortex ( $r=$ $-0.86, p<0.001)$.

\section{DISCUSSION}

Previous studies of prenatal asphyxia after reduction of uterine artery blood flow have not reported on the relationship between the variables monitored and the outcome in terms of either quantitative EEG changes or histology $(9,12)$. This experimental approach should be useful in clarifying the pathophysiology of asphyxia, particularly in view of the absence of the confounding factors of resuscitation and cooling inevitably seen in postnatal studies. In this study, increasing severity of histologic damage was not determined by the degree of hypoxia, but rather by the occurrence of hypotension. This hypotension was of course induced by fetal hypoxia. The most useful predictive factors for parasagittal cortical damage seen during the insult were hypotension, severe flattening of the EEG, and $\mathrm{pH}<7.0$. The most useful criteria, in the first $12 \mathrm{~h}$, were prolonged suppression of the EEG and/or epileptiform activity.

The "watershed" pattern of neuronal damage seen in this study, with damage greatest in the parasagittal cortex, is very similar to that seen in some asphyxiated term infants (13) and in the fetal sheep after transient cerebral ischemia (7). This supports the suggestion that impaired cerebral blood flow is critical in provoking and localizing neuronal injury (14-16). A number of studies of cerebral blood flow during short-term asphyxia have shown consistent regional variations, with reduced cortical flow and preferential brainstem and white matter blood flow despite maintenance of overall blood supply (17-20). This relative reduction of cerebral blood flow to the cortex may explain the increased susceptibility of the parasagittal cortex, even in fetuses who maintained their BP.

It has been suggested that loss of autoregulation means that cerebral flow is essentially pressure passive during asphyxia in the newborn (21). Combined hypoxemia and hypotension lead to a significantly greater fall in cerebral oxygen consumption than hypoxemia alone (22). In this study, the fetal arterial BP during asphyxia was the most powerful predictor of subsequent parasagittal neuronal loss. Although hypotension did not occur in all fetuses that had some neuronal loss, the five fetuses that showed even a transient fall in BP of more than $15 \%$ of baseline had damage. Furthermore, fetuses with no damage actually increased their mean arterial BP during asphyxia. These data point to a primary role for the cardiovascular response to asphyxia in maintaining cerebral homeostasis in utero.

The number of deaths, presumably due to cardiovascular failure, seen in this study emphasizes that the heart is also being asphyxiated. Severe acidosis may impair myocardial contractility $(23,24)$ and, thus, may lead to death even after release of occlusion. Survival with severe brain damage was the least common outcome, even in the relatively controlled conditions of this study. This reflects clinical experience, where compromised fetuses and newborns with severe encephalopathy have a very high mortality (2).

Compared with the pattern of damage seen after transient cerebral ischemia in the fetal sheep (7), in this study there was relatively greater, but patchy, damage to the striatum $(p<$ 
Intensity $(d b)$
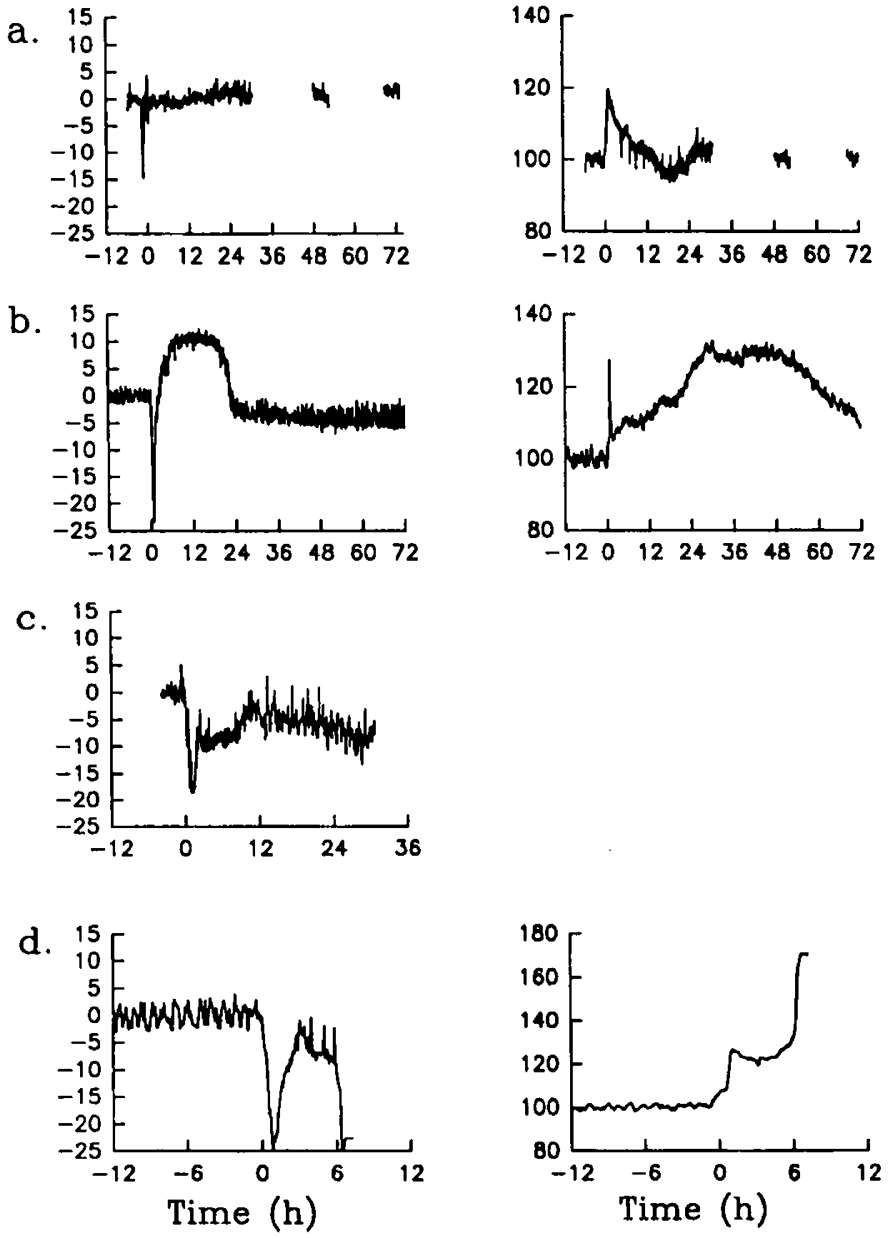

Fig. 3. Examples of different patterns of EEG and impedance changes during and after asphyxia in utero. The asphyxia occurs at time 0 in all cases. $d b$, decibels. $a$, Trivial damage. Despite nearly complete suppression during the asphyxia, the EEG rapidly recovered after release of the occluder. There was a significant increase in impedance during the insult (118\% of baseline), which returned to normal over the subsequent $8 \mathrm{~h}$. For technical reasons, the EEG was able to be recorded continuously only for the first $36 \mathrm{~h}$, with two subsequent $4-\mathrm{h}$ blocks. $b$, Severe damage with early onset seizures. The EEG was more suppressed during the insult, and there was marked hyperactivity beginning within $1 \mathrm{~h}$ postasphyxia, which had resolved to baseline intensity by $24 \mathrm{~h}$. The raw EEG showed intense epileptiform activity, which completely subsided by 48 $\mathrm{h}$. The impedance again showed a rapid rise during the insult, with only partial recovery by $1 \mathrm{~h}$. A marked secondary rise was then seen, peaking $18 \mathrm{~h}$ after the EEG peak, which had not fully resolved by $72 \mathrm{~h}$. $c$, Severe damage, with delayed seizure activity. The impedance was not recorded in this fetus. The EEG remained suppressed ( $<5 \mathrm{db}$ of baseline) for $9 \mathrm{~h}$, then developed hyperactivity (corresponding with epileptiform activity on the raw EEG). The final EEG was very suppressed compared with baseline. $d$. Death. This fetus partially recovered postasphyxia, with a normal $\mathrm{PaO}_{2}$ at $1 \mathrm{~h}$, then developed a progressive deterioration of the EEG and gradual rise of the impedance. When death occurred $5 \mathrm{~h}$ after the insult, the impedance rose dramatically to $180 \%$ of baseline before termination of the recording.

0.0005). In addition, although the overall level of damage in the thalamus was not significantly different, severe focal damage was seen in one fetus that was never found in the ischemic model. These differences may reflect greater susceptibility of the basal ganglia to acidotic insults. Alternatively, asphyxia may be accompanied by differential effects on oxygen delivery to the cerebral regions. During transient cerebral ischemia, there is likely to be consistent residual anastomotic flow to the mid and hind brains
(7). The hippocampus was damaged significantly less often than the parasagittal cortex, which is of particular interest in view of the selective and delayed damage seen in this region after even brief ischemia in the adult rat and mongolian gerbil $(10,25,26)$. This difference must lead to reservations about the relevance of such studies to assessing intervention in the fetus and newborn.

The loss of EEG intensity $3 \mathrm{~d}$ after the insult that occurred with neuronal loss in the underlying parasagittal cortex was similar to that seen after cerebral ischemia in fetal sheep (8). The loss of EEG activity during the insult was a clear marker of the severity of the insult, which suggests that the susceptibility of the brain in vivo is very similar to the situation in vitro, where the outcome of hypoxia is directly proportional to the duration of depolarization $(27,28)$. The rise in cortical impedance reflects cortical energy failure, with neuronal depolarization, and indicates the development of intracellular edema during the insult. As in previous studies of cerebral ischemia in utero, this acute edema was largely reversible (8).

Both delayed epileptiform activity, after a period of EEG suppression, and early onset epileptiform activity were seen in fetuses with neuronal loss. In contrast, only delayed seizures are seen after severe cerebral ischemia in utero (29). This suggests that different mechanisms may be responsible for early compared with delayed seizures. Similar to the ischemia preparation, intense, prolonged epileptiform activity was associated with laminar necrosis in the parasagittal cortex.

The degree of systemic hypoxemia, as measured by the systemic integrated oxygen deficit or the oxygen content, was not related to the outcome, although protocol B, with added inhalational hypoxia, overall produced a more "severe" outcome than protocol $A$. The integrated oxygen deficit to the brain has been shown to be the best indicator of the severity of an insult in isolated brains (30). Our results suggest then that systemic oxygen status does not closely reflect the actual oxygen delivery to the brain, which is a function of both regional cerebral blood flow and oxygen content.

In contrast, the severity of acidosis was closely related to outcome in this study, with a $\mathrm{pH}$ of 7.0 being the cutoff for the presence of significant neuronal loss. Others have suggested that acidosis causes direct neuronal toxicity (5). Two recent studies in vitro, however, have shown that neurons rapidly adapt to local acidosis, and during hypoxia show better survival if the $\mathrm{pH}$ is lowered $(31,32)$. Although these studies may not fully reflect the situation during acute asphyxia, clinical studies of asphyxia neonatorum have also shown that cord blood $\mathrm{pH}$ is a poor predictor of neurologic outcome (33-36). The significance of the systemic base deficit in this study is probably a reflection of the cerebral oxygen deficit, but might also be mediated by cardiac effects as discussed above.

The $50 \%$ rise in blood glucose observed during asphyxia in this study is consistent with previous reports (16). This rise was, however, unrelated to outcome. There is controversy over the influence of glucose levels during hypoxia-ischemia, with beneficial effects suggested with supplementation in neonatal species and deleterious effects in adults (16); specific intervention studies in the fetus are clearly important.

In summary, asphyxia for prolonged periods of up to $2 \mathrm{~h}$ may be accompanied by neuronal death, but not necessarily so in the absence of hypotension or severe acidosis. These data support the suggestion that good cerebral perfusion is critical for surviving asphyxia without damage.

\section{REFERENCES}

1. Levene MI, Kornberg J, Williams THC 1985 The incidence and severity of post asphyxia encephalopathy in fullterm infants. Early Hum Dev 11:21-28

2. Freeman JM (ed) 1985 Prenatal and Perinatal Factors Associated with brain disorders. Washington, DC, NIH 85-1149, pp 237-262

3. Bejar R, Wizniak P, Allard M, Bernirschke K, Baucher Y 1988 Antenatal origin of neurologic damage in newborn infants. Am J Obstet Gynecol 159:357-363 
4. Paul R, Yonekura L, Cantrell C, Turkel S, Pablova Z, Sipos L 1986 Fetal injury prior to labor: does it happen? Am J Obstet Gynecol 154:1187-1193

5. Myers RE, de Courten-Myers GM, Wagner KR 1984 Effect of hypoxia on fetal brain. In: Beard RW, Nathanieisz PW (eds) Fetal Physiology and Medicine, 2nd Ed. Marcel Dekker, New York, pp 419-458

6. Thiringer K, Hrbek A, Karlsson K, Rosen K, Kjellmer I 1987 Postasphyxia cerebral survival in newborn sheep after treatment with oxygen free radical scavengers and a calcium antagonist. Pediatr Res 221:62-66

7. Williams CE, Gunn AJ, Gluckman PD, Synek B 1990 Delayed seizures occurring with hypoxic-ischemic encephalopathy in the fetal sheep. Pediatr Res 27:561-565

8. Williams CE, Gunn AJ, Gluckman PD 1991 Time course of intracellular edema and epileptiform activity following prenatal cerebral ischemia in sheep. Stroke 22:516-521

9. Field DR, Parer JT, Auslender RA, Cheek DB, Baker W, Johnson J 1990 Cerebral oxygen consumption during asphyxia in fetal sheep. J Dev Physiol 14:131-137

10. Smith M, Auer RN, Siesjö BK 1984 The density and distribution of ischemic brain injury in the rat following $2-10 \mathrm{~min}$ of forebrain ischemia. Ann Neuropathol 64:319-332

11. Gasser T, Backer P, Mocks J 1982 Transformations towards the normal distribution of broad band spectral parameters of the EEG. Electroencephalogr Clin Neurophysiol 53:119-124

12. Martin Jr CB, Voermans TM, Jongsma HW 1987 Effect of reducing uteroplacental blood flow on movements and on electrocortical activity of fetal sheep. Gynecol Obstet Invest 23:34-46

13. Hill A, Volpe JJ 1981 Seizures, hypoxic-ischemic brain injury, and intraventricular hemorrhage in the newborn. Ann Neurol 10:109-121

14. Volpe J, Herscovitch P, Perlman JM, Kreusser K, Raichle ME 1985 Positron emission tomography in the asphyxiated term newborn: parasagittal impairment of cerebral blood flow. Ann Neurol 17:287-296

15. Lou HC, Lassen NA, Tweed WA, Johnson G, Jones M, Palahniuk R 1979 Pressure passive cerebral blood flow and breakdown of the blood-brain barrier in experimental fetal asphyxia. Acta Paediatr Scand 68:57-63

16. Vannucci RC 1990 Experimental biology of cerebral hypoxia-ischemia: relation to perinatal brain damage. Pediatr Res 27:317-326

17. Behrman R, Lees M, Peterson E, De Lannoy C, Seeds A 1970 Distribution of the circulation in the normal and asphyxiated fetal primate. Am J Obstet Gynecol 108:956-969

18. Ashwal S, Dale DP, Longo L 1984 Regional cerebral blood flow: studies in the fetal lamb during hypoxia, hypercapnia, acidosis, and hypotension. Pediatr Res 18:1309-1316

19. Laptook A, Stonestreet B, Oh W 1982 The effects of different rates of plas- manate infusions upon brain blood flow after asphyxia and hypotension in newborn piglets. J Pediatr 100:791-796

20. Cavazzuti M, Duffy TE 1982 Regulation of local cerebral blood flow in normal and hypoxic newborn dogs. Ann Neurol 11:247-257

21. Lou HC Lasen NA, Friis-Hansen B 1979 Impaired autoregulation of cerebral blood flow in the distressed newborn infant. J Pediatr 94:118-121

22. Hohimer AR, Chao CR, Bissonette JM 1991 The effect of combined hypoxemia and cephalic hypotension on fetal cerebral blood flow and metabolism J. Cereb Blood Flow Metab 11:99-105

23. Cabal LA, Devaskar U, Siassi B, Hodgman HE 1984 Cardiogenic shock associated with perinatal asphyxia in preterm infants. Stroke 15:1029-1032

24. Davies JM, Tweed WA 1984 The regional distribution and determinants of myocardial blood flow during asphyxia in the fetal lamb. Pediatr Res 18:764767

25. Tyson GW, Teasdale GW, Graham DI, McCulloch J 1984 Focal cerebral ischemia in the rat: topography of hemodynamic and histopathological changes. Ann Neurol 15:559-564

26. Kirino T 1982 Delayed neuronal death in the gerbil hippocampus following ischemia. Brain Res 239:57-69

27. Somjen GG, Aitken PG, Balestrino M, Herreras O, Kawasaki K 1990 Spreading depression-like depolarization and selective vulnerability of neurons. A brief review. Stroke 2:III-179-III-183

28. Siesjö BK, Bengtsson A 1989 Calcium fluxes, calcium antagonists, and calciumrelated pathology in brain ischemia, hypoglycemia, and spreading depression: a unifying hypothesis. J Cereb Blood Flow Metab 9:127-140

29. Williams CE, Gunn AJ, Mallard EC, Gluckman PD 1992 Outcome after ischemia in the developing sheep brain: an electroencephalographic and histological study. Ann Neurol 31:14-21

30. Emoto SE, Kintner D, Feyzi JM, Gilboe DD 1988 Relating cerebral ischemia and hypoxia to insult intensity. J Neurochem 50:1952-1958

31. Sher PK 1990 The effects of acidosis on chronically hypoxic neurons in culture. Exp Neurol 107:256-262

32. Giffard RG, Monyer H, Christine CW, Choi DW 1990 Acidosis reduces NMDA receptor activation, glutamate neurotoxicity, and oxygen-glucose deprivation neuronal injury in cortical cultures. Brain Res 506:339-342

33. Gilstrap LC, Leveno KJ, Burris J, Williams ML, Little BB 1989 Diagnosis of birth asphyxia on the basis of fetal $\mathrm{pH}$, Apgar score, and newborn cerebral dysfunction. Am J Obstet Gynecol 161:825-830

34. Ruth VJ, Raivio KO 1988 Perinatal brain damage: predictive value of metabolic acidosis and the Apgar score. Br Med J 297:24-37

35. Dijxhoorn M, Visser G, Fidler V, Touwen BCL, Huisjes H 1986 Apgar score, meconium and acidaemia at birth in relation to neonatal neurological morbidity. Br J Obstet Gynaecol 93:217-222

36. Lauener PA, Calame A, Janacek P, Bossart H, Monod JF 1983 Systematic $\mathrm{pH}$-measurements in the umbilical cord: cause and predictive value of neonatal acidosis. J Perinat Med 11:278-285 